\title{
The Generic Beamline Concept of the PETRA III Undulator Beamlines
}

\author{
U. Hahn, H. B. Peters, R. Röhlsberger, and H. Schulte-Schrepping \\ Deutsches Elektronen-Synchrotron DESY, Notkestrasse 85, 22603 Hamburg, Germany
}

\begin{abstract}
The conversion of the PETRA storage ring at DESY to a third generation synchrotron radiation light source [1] poses a challenge to the design of the beam transport system. The total power in the white beam will be as high as $7.5 \mathrm{~kW}$ in the case of the $5 \mathrm{~m}$ long undulator at $100 \mathrm{~mA}$. The power density will be $476 \mathrm{~W} / \mathrm{mm}^{2}$ at $20 \mathrm{~m}$ from the source. Upgrades to a beam current of $200 \mathrm{~mA}$ have to be accounted for in the design of the beamline components. For the beam transport between the undulator and the experimental hall, the design of a generic beamline is presented. It contains all elements which are needed to guide the beam to the experiment. This generic beamline consists of the estimated maximum of components for this purpose. Special experimental needs may reduce the number of proposed devices in the generic part and add special optical devices close to the experiment, e. g. strong focusing. The paper focuses on the girder concept for all major beam transport components and the collimating shutter system which has to deal with the high power density of the PETRA III undulators.
\end{abstract}

Keywords: PETRA III, Synchrotron Radiation, Generic Beamlines, front end, high heat load PACS: 07.85.Qe, 41.50.+h

\section{INTRODUCTION}

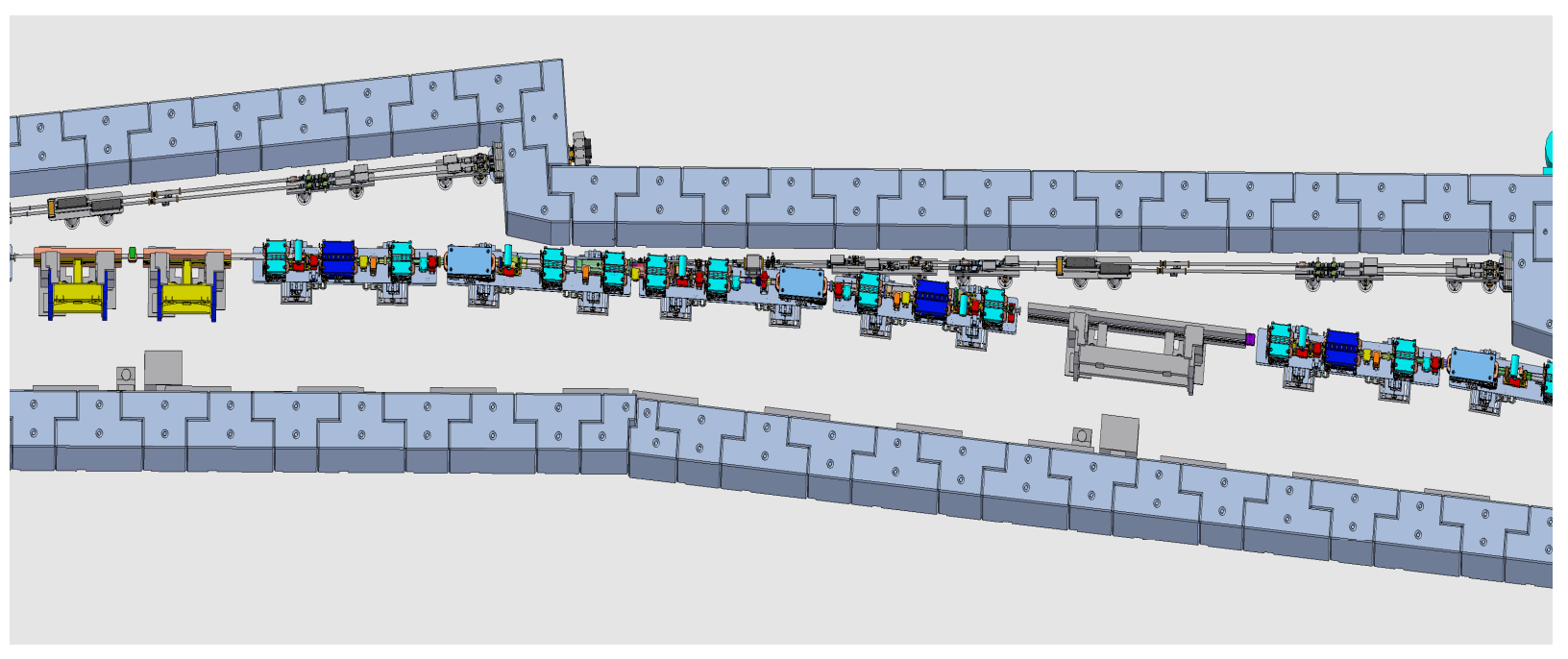

FIGURE 1. A view into the new PETRA III tunnel section with two canted undulators on the left and their beamline front ends

Beginning in 2007 DESY will rebuild the $2304 \mathrm{~m}$ circumference storage ring PETRA II into the 3rd generation synchrotron radiation source PETRA III[1] with the particle energy of $6 \mathrm{GeV}$, a beam emittance of $1 \mathrm{nmrad}$, and an initial current of $100 \mathrm{~mA}$ in top-up operation. 14 independent undulators will deliver highly brilliant beams for experiments. Four of the undulators are $5 \mathrm{~m}$ long single devices. The other ten are $2 \mathrm{~m}$ long. Pairs of the $2 \mathrm{~m}$ long devices are arranged before and after a 5 mrad deflection of the electron beam in the $5 \mathrm{~m}$ long straight sections 
(canted undulators). For the beam transport between the undulator and the experimental hall a generic beamline is designed that contains all the elements which are needed to guide the beam to the experiment. In Fig. 1 a PETRA III tunnel segment with the undulator and the beamline front end is shown.

The main tasks of the generic beamline are the following:

- $\quad$ provide a hydrocarbon and dust free vacuum system connected windowless to the storage ring

- transport the photon beam from the undulator to the experiment, conserving the beam properties in spite of the high power density

- $\quad$ shape the beam with fixed and movable masks, reduce the power load on optical components

- $\quad$ ensure radiation safety by collimation and suitable beam stops

- $\quad$ ensure equipment protection by an appropriate vacuum interlock system

- give access to the experiment conserving the thermal balance of the optical components

- monitor the photon beam position

- filter the white beam to reduce the power load and suppress harmonics

- monochromatize the white beam

- collimate or focus the white or monochromatic beam

The layout is split into two parts separated by the storage ring tunnel wall. Optical components, such as mirrors and monochromators, will be placed outside the ring tunnel. Special experimental needs may reduce the number of devices in the generic part and add special optical devices in the storage ring tunnel or close to the experiment.

\section{GENERIC BEAMLINE INSTALLATIONS}

Inside the ring tunnel all components in the photon beamline dealing with radiation safety, beam position and beam collimation will be placed as shown in Fig. 2. There will be the option for a collimating lens system installed inside the ring tunnel.

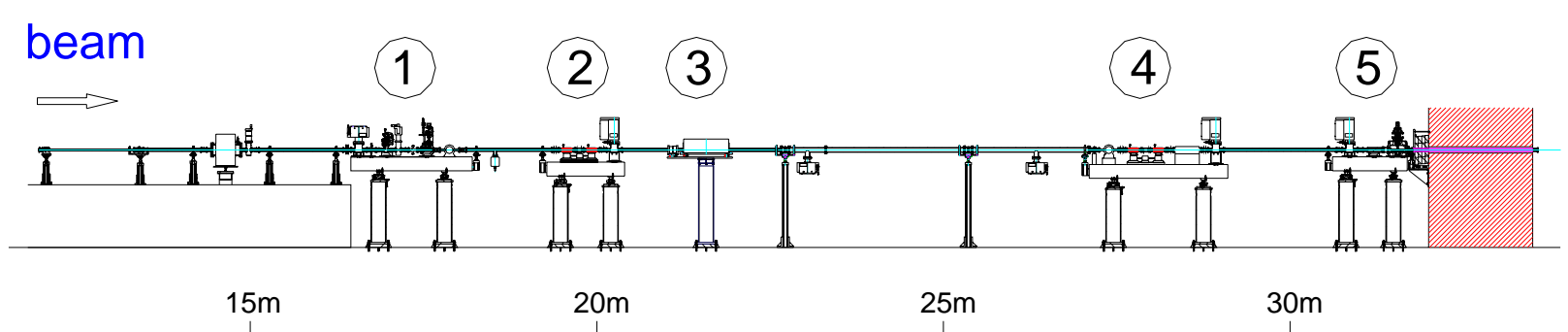

tunnel section

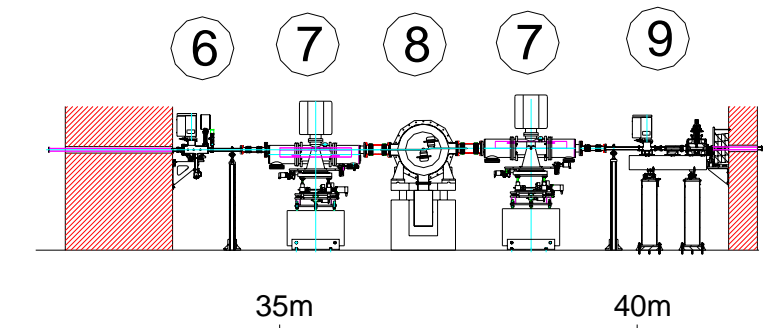

1 granite girder for valves and X-ray BPM

2 primary collimating slit

3 permanent magnet for radiation safety

4 secondary collimating slit and photon shutter

5 bremsstrahlung collimator and beam shutter

6 vacuum diagnostic chamber

7 mirror chamber

8 monochromator

first optics hutch

9 bremsstrahlung stop and secondary beam shutter

FIGURE 2. The generic beamline layout for a single beamline. The scale measures the distance from the undulator source.

PETRA III will run in top-up mode which requires a special arrangement of radiation safety components. For a fail safe particle injection, each beam outlet has to be equipped with a permanent magnet which extracts misguided positrons out of the photon beamline. The beam shutter will be placed directly in front of the storage ring wall. A 
photon shutter absorbing the photon beam power protects the beam shutter from melting. To limit the neutron dose caused by the bremsstrahlung originating from positron beam losses inside the undulator vacuum chamber, two vertical collimating absorber slits are installed in the beamline at $20 \mathrm{~m}$ and $28 \mathrm{~m}$ from the source.

Space is also needed for the vacuum protection system, i.e. the fast acting valve. The vacuum failure sensing devices will be installed in the first optics hutch. The windowless beam transport system will connect the storage ring and the first optics hutch. The high heat load monochromators, optional mirror chambers and bremsstrahlung stops with secondary beamshutters are placed inside this hutch.

In the following the girder concept for all major beam transport components and the collimating slit and shutter system which has to deal with the high power density of the PETRA III undulator are described.

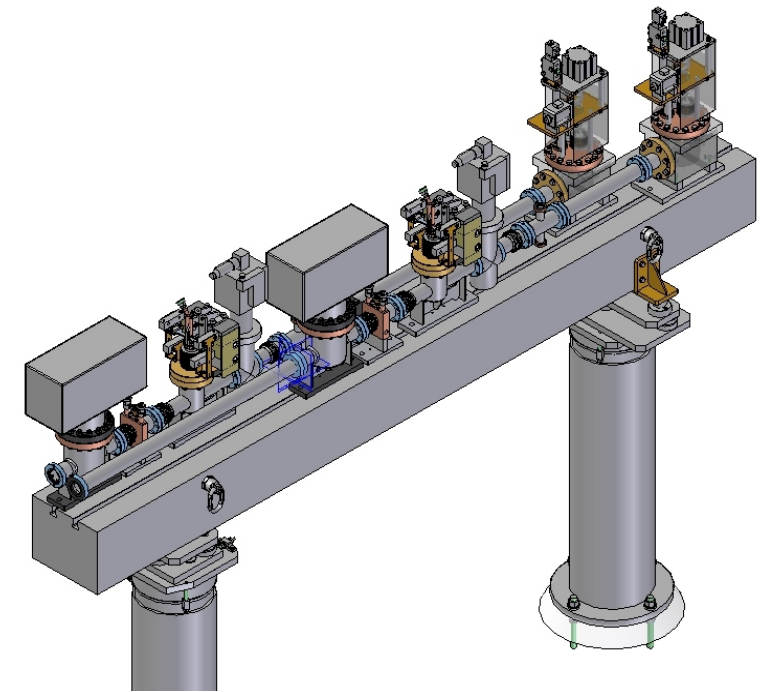

FIGURE 3. The first granite girder of the canted undulator beamline

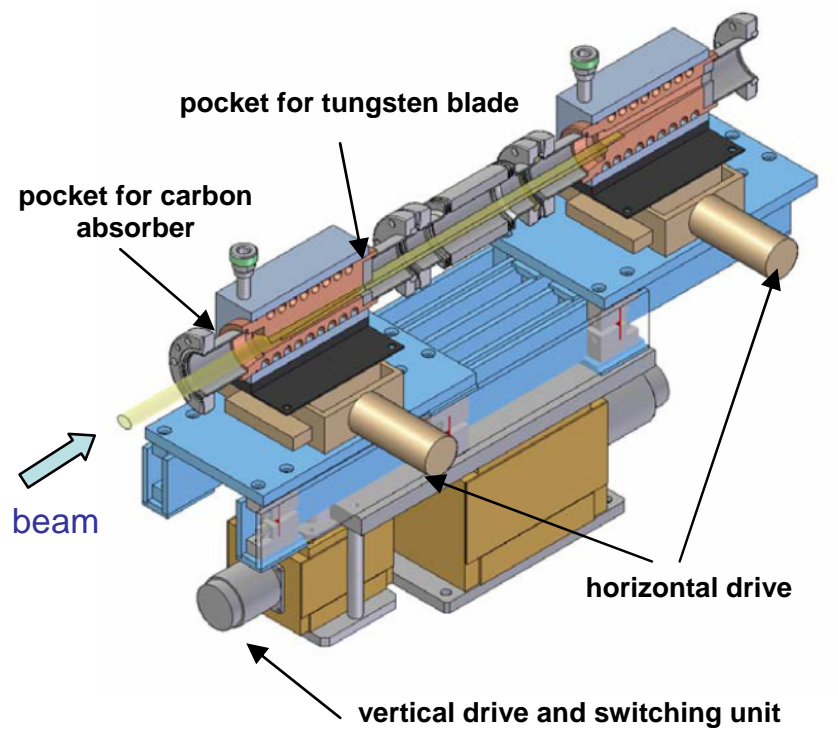

FIGURE 4: High power slit system and photon shutter

\section{The Girder Concept}

There is limited space in the PETRA III storage ring tunnel. The distance to the tunnel wall is $50 \mathrm{~cm}$ only. Because of the large PETRA III bending radius and the small bending angle between the straight sections, there is only a small separation between photon beamlines and the machine. Additionally, the 10 canted beamlines with their angular separation of 5mrad are close together. Therefore all major beamline components in the storage ring tunnel will be placed, aligned and fixed with granite girders (see Figs. 2 and 3 ) before installation in the storage ring tunnel. Precision $\mathrm{T}$ - slots on the granite girders allow to align and to fix the components supported by the girder in a reproducible way. These slots guarantee an exact alignment of the installed components to the beamline axis. There are several suppliers for such granite beams with high precision (flatness $<10 \mu \mathrm{m}$, straightness of the slot $<10 \mu \mathrm{m})$. On the girder the beamline components are aligned to each other very precisely. This is especially advantageous for the canted beamlines with the $5 \mathrm{mrad}$ horizontal angle between the beamlines. The girders are supported with a three point kinematic mount system on two sand filled steel columns. The kinematical mount uses precision balls as supporting element with the same size as the retro reflectors of the laser tracker system used for the basic alignment. This allows aligning of the support system with high precision. The girder system allows a pre-alignment of the supported beamline components outside the storage ring tunnel with high precision. In the case of maintenance, the positioning and replacement of a whole girder, using backup systems, reduces the setup time in the storage ring tunnel. 


\section{High Power Slit and Photon Shutter System}

There exist several concepts for high power slit systems [2, 3, 4]. The design of the PETRA III slit systems is shown in Fig. 4. The slits collimate the beam by absorbing the off axis radiation of the undulator. The second slit system additionally acts as a power absorber to shut off the photon beam [4]. While the first slit collimates only in the vertical direction, the second slit also collimates in the horizontal direction. Figure 4 shows a vertical cut through the slit jaws and the principle of operation of the whole shutter - slit system [5]. The upper and lower jaws consist of an inclined water cooled absorber which accepts the beam power. The absorber material is GlidCop ${ }^{\circledR}$. The cooling channels are machined to wind radially around the central copper block. This absorber block finally is brazed into a stainless steel body connected to the support system. Pockets in front and behind the jaws allow dissipating the low energetic radiation into carbon, whereas a tungsten blade behind the jaw will stop most of the high energetic radiation to reduce the background radiation in the experimental area. The slit height is controlled by one drive moving both jaws. The vertical slit position is adjusted by moving the whole assembly up and down. The shutter function of the slit is achieved by a pneumatic cylinder incorporated in the vertical slit drive. The horizontal slit function will be accomplished by integrating a horizontal movable absorber jaw [3].

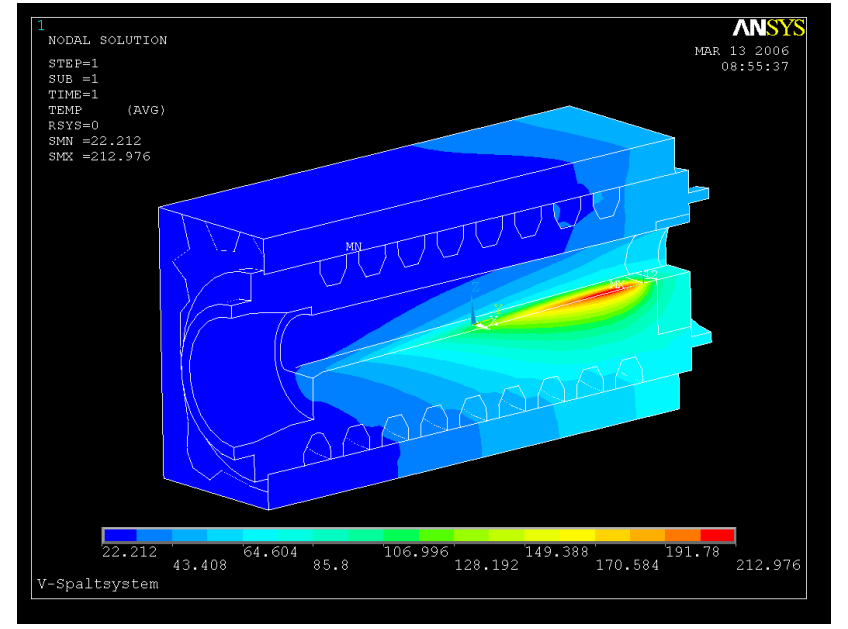

FIGURE 5. Calculation of the typical thermal distribution of the absorbed undulator beam at the first vertical collimating slit jaw.

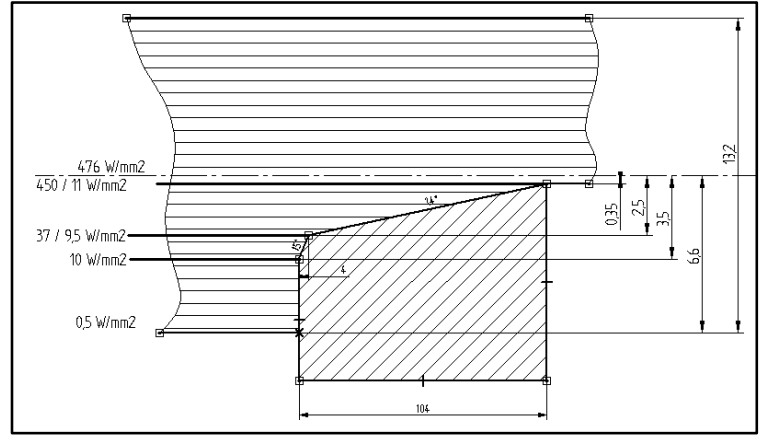

FIGURE 6. Cut through the slit jaw and the vertical power distribution, showing the shape with two different angles related to the reduced power load at the border of the beam.

The total power in the white beam of the $5 \mathrm{~m}$ undulator at $100 \mathrm{~mA}$ will be $7.5 \mathrm{~kW}$ with a maximum power density of $476 \mathrm{~W} / \mathrm{mm}^{2}$ at $20 \mathrm{~m}$ distance from the source.

Figure 5 shows a single slit jaw shaping the beam vertically at 20m. FEM model calculations (ANSYS) with a typical power load on a single slit jaw at an inclination angle of $1.4^{\circ}$ have been performed. A vertical cut through the power distribution of the beam and the slit jaw is shown in Fig. 6 . In this case the single jaw absorbs $\sim 40 \%$ of the total power at $20 \mathrm{~m}$ distance from the source. The calculations for a current of $100 \mathrm{~mA}$ show, that the beam shaping with the slit system will cause a maximum temperature of $213^{\circ} \mathrm{C}$ at a water-cooling temperature of $20^{\circ} \mathrm{C}$. The $2 \mathrm{~m}$ long canted devices will roughly deliver the same power at $200 \mathrm{~mA}$ beam current. The possibility to install graphite absorber plates in the pockets in front of the slit jaws will reduce the power load on the slits and give flexibility for higher beam currents at the $5 \mathrm{~m}$ and $20 \mathrm{~m}$ long devices.

\section{REFERENCES}

1. PETRAIII: “A low Emittance Synchrotron Radiation Source”, Technical Design Report DESY 2004 - 035, 211 - 218

2. T. Mochizuki, S. Takahashi, and H. Kitamura, "Front-end combination component of fixed mask and absorber", AIP Conference Proceedings SRI 2003, (2004) 705, 474 - 477

3. P. Marion and L. Zhang, "High power primary slits for the ESRF Beamlines”, AIP Conference Proceedings SRI 2003, (2004)

4. C. Benson et al., "Mechanical design of a front end for canted undulators at the Advance Photon Source”, AIP Conference Proceedings SRI 2003, (2004) 705, 474 - 477

5. U. Hahn and M. Rueter, „Hochleistungsstrahlverschluss - und Spaltsystem fuer Synchrotronstrahlung“, German Patent Nr. DE 10135307 C2 